\title{
A Case of Fallopian Tube Metastasis from Cervical Adenocarcinoma
}

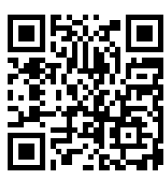

\author{
Manoharan $\mathbf{M}^{1}$ and KarikalanB ${ }^{2 *}$ \\ ${ }^{1}$ Department of Pathology, Gribbles pathology, Malaysia \\ ${ }^{2}$ Department of Pathology, Perdana University, Malaysia
}

Received: April 09, 2018; Published: April 17, 2018

*Corresponding author: Barani Karikalan, Department of Pathology, Perdana University, Malaysia, Tel: 60108148824; Email: baranisri@gmail.com

\section{Introduction}

Invasive endocervical adenocarcinomas, though microinvasive, have the ability to metastasize to the ovaries by retrograde transtubal spread. Endocervical adenocarcinomas with ovarian metastases simulating primary ovarian surface epithelial neoplasms have been reported infrequently. Here, we present a case of adenocarcinoma involving the endocervix and a fallopian tube. The tubectomy might be a possible explanation for the absence of ovarian or peritoneal spread of the tumour.

\section{Case Report}

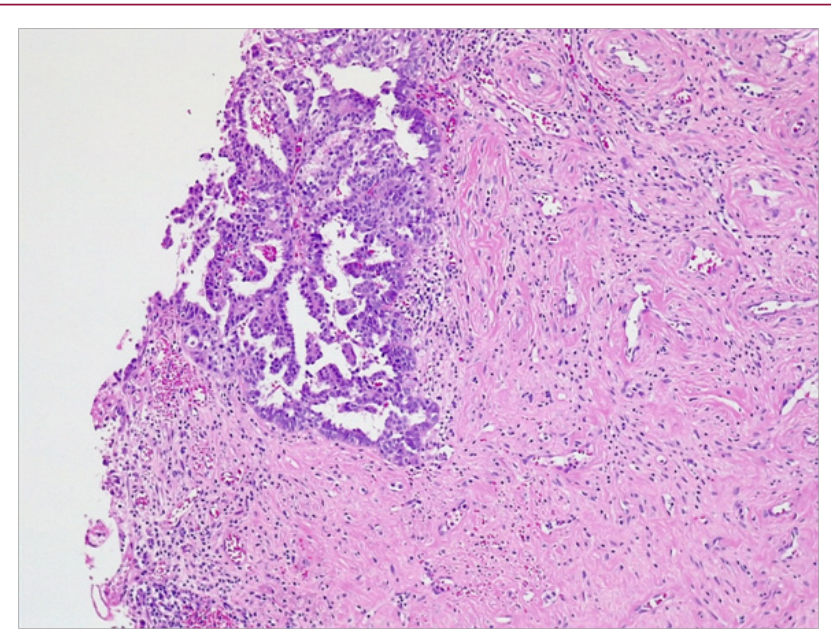

Figure 1: The Endocervix Shows a Focus of Adenocarcinoma.

A 57-year-old female underwent transabdominal hysterectomy with bilateral salpingoophorectomy for adenocarcinoma of the cervix diagnosed by cervical biopsy. On gross examination, the cervix appeared irregular and ulcerated. The endometrium was thinned out and myometrium measured $20 \mathrm{~mm}$ in maximum thickness. The fallopian tubes showed features of tubectomy. In addition, the right tube was distended measuring $50 \times 15 \mathrm{~mm}$. Cut section showed solid and grey white tumour occupying the lumen. Left fallopian tube and both ovaries were within normal limits. Microscopically, the cervix showed extensive ulceration. Only a tiny focus of remnant of adenocarcinoma was seen, possibly from the earlier surgical intervention. The lesion showed papillary pattern formed of multilayered cuboidal to columnar cells exhibiting pleomorphism, scanty cytoplasm and increased mitosis (Figure 1). The uterine wall showed atrophic endometrium with extensive areas of ulceration. No tumor was present (Figure 2). The right fallopian tube showed dilated lumen filled with adenocarcinoma exhibiting complex papillary and solid pattern. Individual tumor cells were cuboidal to low columnar with scant cytoplasm and increased mitosis. The tumor was confined to the mucosa. The mucosa adjacent to the tumor showed no carcinoma in situ changes (Figures 3-6). The left fallopian tube and ovaries were within normal limits (Figures 7 \& 8).

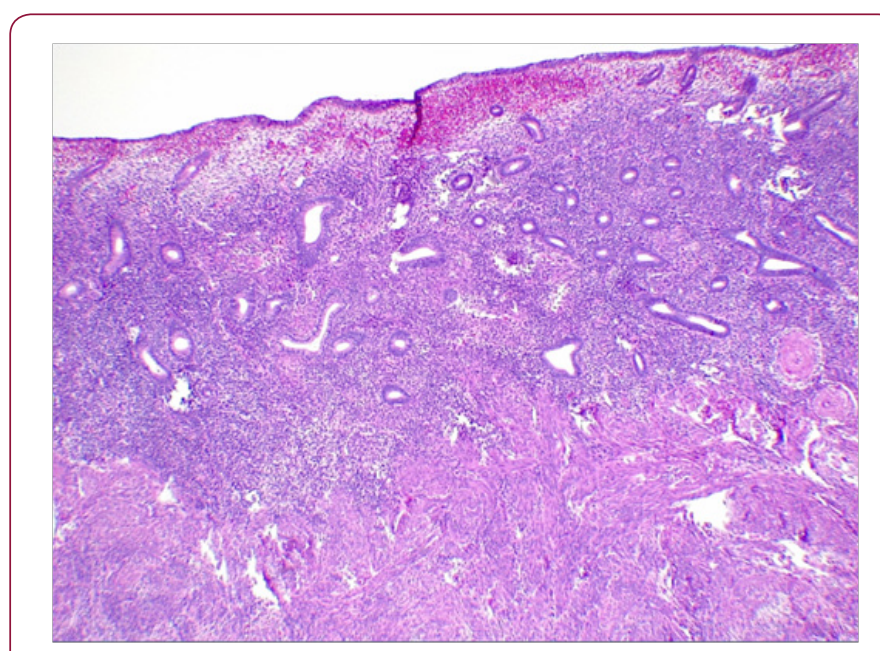

Figure 2: Endometrium is Atrophic. 


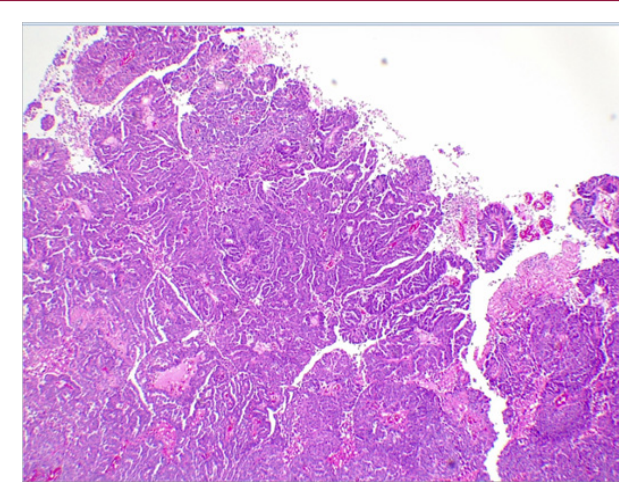

Figure 3: Fallopian Tube is filled with Carcinoma.

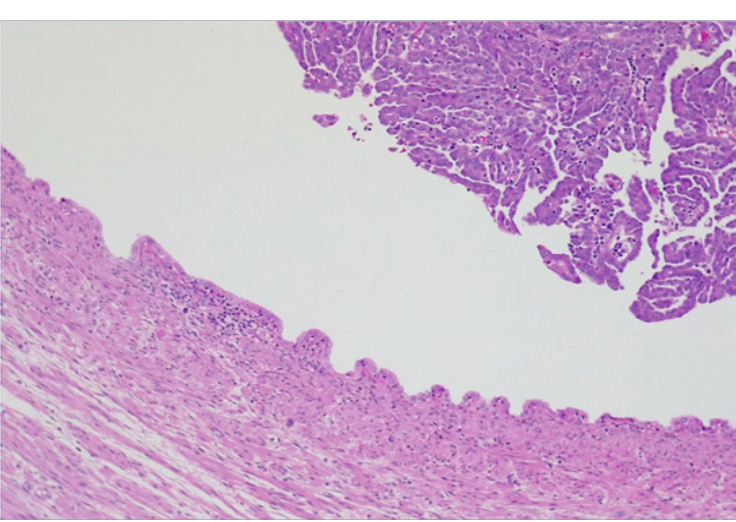

Figure 4: Fallopian Tube is Filled with Carcinoma. The Adjacent Mucosa Shows no in Situ Changes.

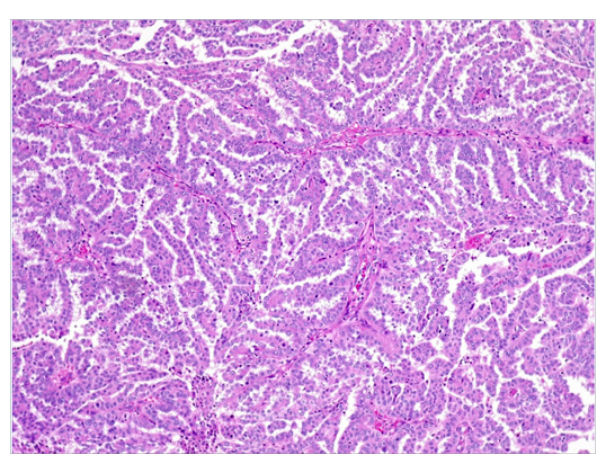

Figure 5: Carcinoma Arranged as Slit Like Spaces and Papillary Process.

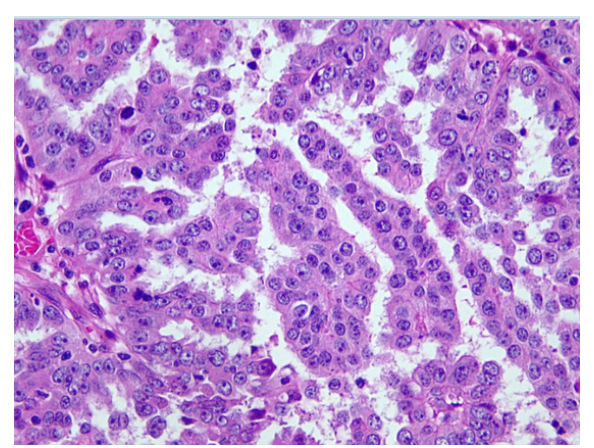

Figure 6: Carcinoma Arranged as Slit Like Spaces and Papillary Process.

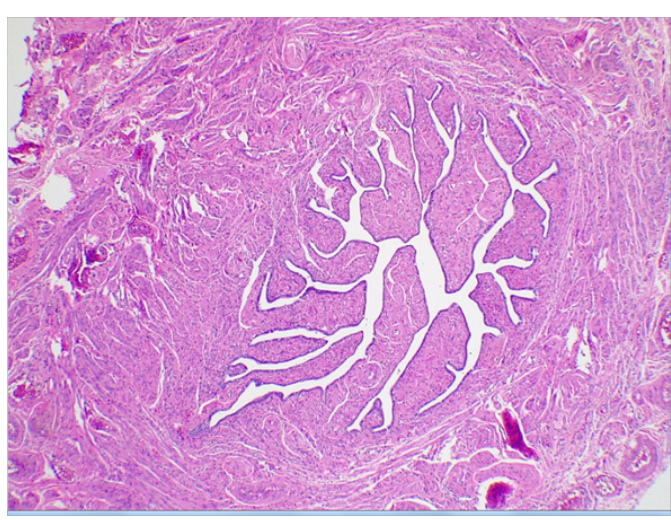

Figure 7: Other Fallopian Tube is Free of Tumour.

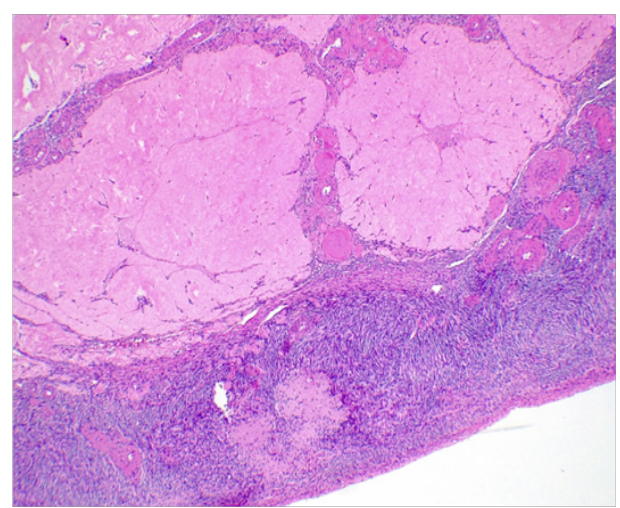

Figure 8: Ovary is Free of Tumour.

\section{Discussion}

According to SEER registry, there are 7.7 new cases of cervical cancer per 100,000 women per year. Improved Pap smear screening has resulted in steady decrease in the number of new cases of cervical cancer over the past decade. But this decrease is limited to squamous cell carcinoma of the cervix only. The incidence of adenocarcinoma of the cervix seems to be rising, especially in women of younger age group [1-3]. Factors like obesity, nulliparity, trends in oral contraceptive usage and difficulty in diagnosing glandular lesions by Pap smear are suggested as reasons [1]. Very few cases of cervical carcinoma involving the upper genital tract have been reported in the literature [4,5]. Increased sampling of fallopian tubes due to change in thoughts about genesis of ovarian tumors has contributed to the increase in number of fallopian tube cancers reported. Rabban et al. [6] studied metastatic fallopian cancers from 100 non gynecological cancers.

Most are adenocarcinomas from breast and colon favoring fimbriae of the fallopian tube and grow as flat to exophytic lesions. They often tend to resemble primary fallopian tube cancers leading to diagnostic difficulty and sometimes require ancillary HPV testing to confirm diagnosis [6,7]. Fallopian tube metastasis from cervical carcinoma is extremely rare and the reported cases are mainly of squamous cell carcinoma [8]. Reyes et al. [9] studied 20 cases of uterine corpus and adnexa involvement by cervical carcinoma. Eight out of ten endocervical adenocarcinomas showed fallopian 
metastasis [9]. The lesions resembled primary fallopian tube carcinoma. Distinct morphologic analysis and immunohistochemical features are suggested to distinguish metastatic adenocarcinoma from primary tubal malignancies [9]. Over expression of p53 is seen in tubal serous adenocarcinomas while negative p53 indicate endocervical adenocarcinoma.

Tubal serous neoplasm is also positive for WT-1 [10]. The current paradigm of fallopian tube being the origin of serous carcinomas of the ovary has led to increase of fallopian tube sampling, which may have led to increased recognition of metastatic fallopian tube cancers. Thus, all metastatic fallopian tube carcinomas arising from both gynecological and non-gynecological malignancies have recently gained attention $[6,11,12]$. In our case, morphologic pattern suggest the spread of primary cervical adenocarcinoma to the fallopian tube. Contiguous and transtubal spread is the proposed mechanism of spread of cervical carcinoma to the upper genital tract [10]. Fallopian tube being involved, prognosis and treatment remain unclear with a major concern for tumor spillage into the peritoneal cavity. FIGO staging does not clearly indicate the clinical staging of endocervical carcinoma involving the fallopian tube [13]. The hypothesis of tubal ligation impeding transtubal spread of cancer cells from lower female genital tract has been tested. Lower stage and less mortality was seen in aggressive endometrial carcinoma patients with tubal ligation suggesting the importance of transtubal spread [14]. In conclusion, involvement of upper genital tract by cervical adenocarcinoma is rare. This case is one example, also indicating the impact of tubal ligation on tumor spread.

\section{References}

1. (1996) SEER Stat Fact Sheets. Cervix Uteri Cancer. SEER: Surveillance, Epidemiology, and End Results Program [database online]. Bethesda: National Cancer Institute, USA.

2. Wang SS, Sherman ME, Hildesheim A, Lacey JV, Devesa S (2004) Cervical adenocarcinoma and squamous cell carcinoma incidence trends among white women and black women in the United States for 1976-2000. Cancer 100(5): 1035-1044.

3. Smith HO, Tiffany MF, Qualls CR, Key CR (2000) The rising incidence of adenocarcinoma relative to squamous cell carcinoma of the uterine cervix in the United States-A 24-year population-based study. Gynecol Oncol 78: 97-105.

4. Gungor T, Altinkaya SO, Ozat M, Akbay S, Mollamahmutoglu L (2011) Unusual form of superficial spreading squamous cell carcinoma of cervix involving the endometrium, bilateral tubes and ovaries: a case report with literature review. Arch Gynecol Obstet 283(2): 323-327.

5. Jaiman S, Surampudi K, Gundabattula SR, Garg D (2014) Bilateral ovarian metastatic squamous cell carcinoma arising from the uterine cervix and eluding the Mullerian mucosa. Diagn Pathol 9: 109.

6. Rabban JT, Vohra P, Zaloudek CJ (2015) Nongynecologic metastases to fallopian tube mucosa: a potential mimic of tubal high-grade serous carcinoma and benign tubal mucinous metaplasia or nonmucinous hyperplasia. Am J Surg Pathol 39(1): 35-51.

7. Ronnett BM, Yemelyanova AV, Vang R, Gilks CB, Miller D, et al. (2008) Endocervical adenocarcinomas with ovarian metastases: analysis of 29 cases with emphasis on minimally invasive cervical tumors and the ability of the metastases to simulate primary ovarian neoplasms. Am J Surg Pathol 32(12): 1835-1853.

8. Gungor T, Altinkaya SO, Ozat M, Akbay S, Mollamahmutoglu L (2011) Unusual form of superficial spreading squamous cell carcinoma of cervix involving the endometrium, bilateral tubes and ovaries: a case report with literature review. Arch Gynecol Obstet 283(2): 323-327.

9. Reyes C, Murali R, Park KJ (2015) Secondary involvement of the adnexa and uterine corpus by carcinomas of the uterine cervix. A detailed morphologic description. Int J Gynecol Pathol 34(6): 551-563.

10. Hashi A, Yuminamochi T, Murata S, Iwamoto H, Honda T (2003) Wilms tumor gene immunoreactivity in primary serous carcinomas of the fallopian tube, ovary, endometrium and peritoneum. Int J Gynecologic Pathol 22(4): 374-377.

11. Crum CP (2009) Intercepting pelvic cancer in the distal fallopian tube: Theories and realities. Mol Oncol 3(2): 165-170.

12. Singh N, Gilks CB, Wilkinson N, McCluggage WG (2015) Assessment of a new system for primary site assignment in high-grade serous carcinoma of the fallopian tube, ovary, and peritoneum. Histopathology 67(3): 331337.

13. (2013) Protocol for the Examination of Specimens from Patients with Carcinoma of the Uterine Cervix. College of American Pathologists Cancer Protocol Templates.

14. Ashley S, Louise A, Scott D, David E, et al. (2015) Relationships of Tubal Ligation to Endometrial Carcinoma Stage and Mortality in the NRG Oncology/ Gynecologic Oncology Group 210 Trial. JNCI J Natl Cancer Inst 107(9): 158.

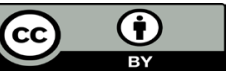

This work is licensed under Creative Commons Attribution 4.0 License

Submission Link: https://biomedres.us/submit-manuscript.php

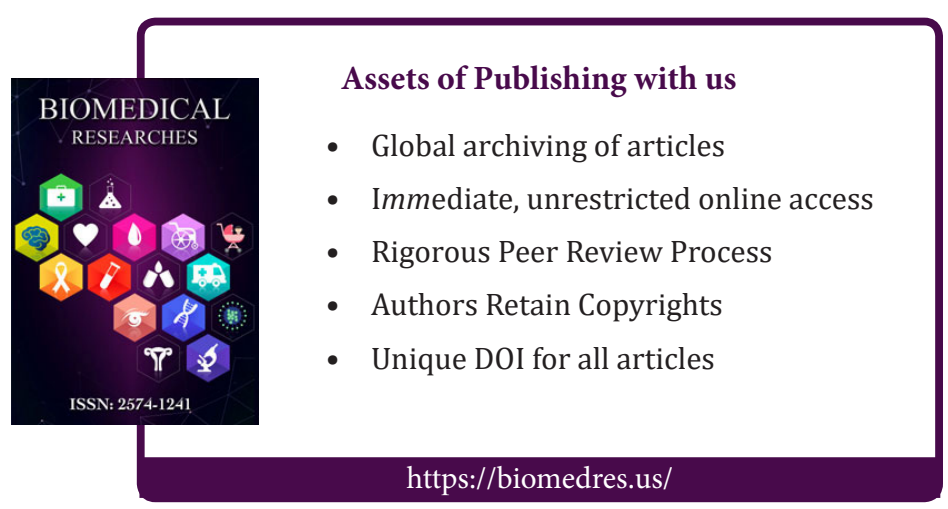

